\title{
The HIV protease inhibitor ritonavir blocks osteoclastogenesis and function by impairing RANKL-induced signaling
}

Michael W.-H. Wang, ${ }^{1}$ Shi Wei, ${ }^{1}$ Roberta Faccio, ${ }^{1}$ Sunao Takeshita, ${ }^{1}$ Pablo Tebas, ${ }^{2}$ William G. Powderly, ${ }^{2}$ Steven L. Teitelbaum, ${ }^{1}$ and F. Patrick Ross ${ }^{1}$

${ }^{1}$ Department of Pathology and Immunology and 'Department of Medicine, Washington University School of Medicine, St. Louis, Missouri, USA.

\begin{abstract}
Highly active antiretroviral therapy (HAART), which includes HIV protease inhibitors (PIs), has been associated with bone demineralization. To determine if this complication reflects accelerated resorptive activity, we studied the impact of two common HIV PIs, ritonavir and indinavir, on osteoclast formation and function. Surprisingly, we find that ritonavir, but not indinavir, inhibits osteoclast differentiation in a reversible manner and also abrogates bone resorption by disrupting the osteoclast cytoskeleton, without affecting cell number. Ritonavir given in vivo completely blunts parathyroid hormone-induced osteoclastogenesis in mice, which confirms that the drug is bone sparing. In keeping with its antiresorptive properties, ritonavir impairs receptor activator of nuclear factor $\kappa B$ ligand-induced (RANKL-induced) activation of NF- $\kappa B$ and Akt signaling pathways, both critical to osteoclast formation and function. In particular, ritonavir is found to inhibit RANKL-induced Akt signaling by disrupting the recruitment of TNF receptor-associated factor 6/c-Src complex to lipid rafts. Thus, ritonavir may represent a bone-sparing PI capable of preventing development of osteopenia in patients currently on HAART.
\end{abstract}

\section{Introduction}

Throughout life, bone remodeling occurs by a finely orchestrated process of osteoclastic resorption and osteoblastic formation. When intact, this process ensures maintenance of skeletal integrity and calcium homeostasis. In all circumstances bone loss occurs when the activity of the resorptive cell exceeds that of its anabolic counterpart. For example, postmenopausal osteoporosis is caused by an absolute increase of osteoclasts and osteoblasts, with the former activity outpacing that of the latter (1). Senile (type II) osteoporosis, in contrast, is a low-turnover disease, but once again bone resorptive activity exceeds that of matrix deposition and calcification (1). Therefore, one approach to dissecting the cause of bone loss in a specific clinical circumstance is to examine the direct effects of various drugs on generation and activity of osteoclasts and osteoblasts.

Osteoclasts are multinucleated cells generated by the fusion of mononuclear progenitors of the monocyte/macrophage family (2). The pathway involved in osteoclast differentiation and activation requires two key elements: receptor activator of nuclear factor $\mathrm{KB}$ (RANK), found in osteoclasts and their precursors, and RANK ligand (RANKL), produced by osteoblasts and stromal cells in the bone marrow $(2,3)$. In addition, M-CSF is required for survival and proliferation of osteoclast precursors. Ligation of RANKL to RANK on macrophages prompts selective intracellular signals that eventuate in the assumption of the osteoclast phenotype (4).

Nonstandard abbreviations used: constitutively active (CA); electrophoretic mobility shift assay (EMSA); extracellular signal-related kinase (ERK); highly active antiretroviral therapy (HAART); parathyroid hormone (PTH); protease inhibitor (PI); RANK ligand (RANKL); receptor activator of nuclear factor $\kappa \mathrm{B}$ (RANK); tartrate-resistant acid phosphatase (TRAP); TNF receptor-associated factor 6 (TRAF6).

Conflict of interest: The authors have declared that no conflict of interest exists.

Citation for this article: J. Clin. Invest. 114:206-213 (2004)

doi:10.1172/JCI200415797.
Bone loss is a recently described clinical condition in HIVinfected patients on highly active antiretroviral therapy (HAART). Prior to the introduction of HAART, HIV-infected adults generally exhibited normal bone mineral density, which remained stable with time (5). While one component of HAART, namely HIV protease inhibitors (PIs), are candidate osteopenic agents (6-9), a firm link between this family of drugs and bone loss remains to be established.

To address this issue we examined the effects of two PIs on osteoblast and osteoclast function. In keeping with the loss of bone experienced by HAART-treated patients on PI, indinavir attenuates osteoblast recruitment and the capacity of these cells to synthesize bone (10). Surprisingly, however, another PI, ritonavir, while not impacting osteoblasts, exerts similar repressive effects on the osteoclast. Formation and activation of osteoclasts is mediated primarily by the activity of the unique cytokine RANKL (11). We have demonstrated previously that exposure of osteoclasts or their precursors to IL-4, a molecule that inhibits osteoclastogenesis and function, blocks several major RANKL-stimulated signaling pathways (12). Given these observations, we examined the impact of ritonavir on these events and find that the PI selectively inhibits NF- $\mathrm{KB}$ and Akt signaling stimulated by the cytokine.

\section{Results}

Ritonavir inhibits osteoclastogenesis in vitro. To determine the effects of PIs on osteoclastogenesis, we turned to pure (>99\%) populations of bone marrow macrophages that, in the presence of M-CSF and RANKL, differentiate into multinucleated cells phenotypically and functionally indistinguishable from authentic osteoclasts. While addition of the osteoblast-inhibiting PI indinavir (10) does not impact the osteoclastogenic process, ritonavir dose dependently impairs osteoclast formation with an $\mathrm{IC}_{50}$ of approximately $10 \mu \mathrm{g} / \mathrm{ml}$ (Figure 1 , A and B). Reflecting 


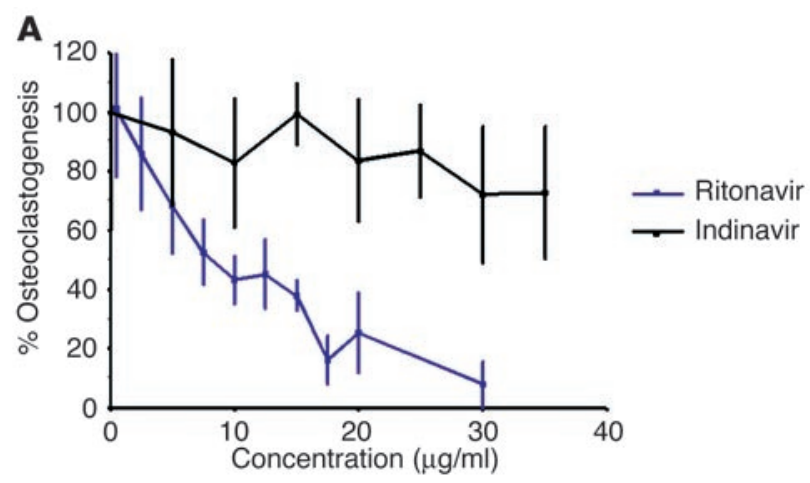

B

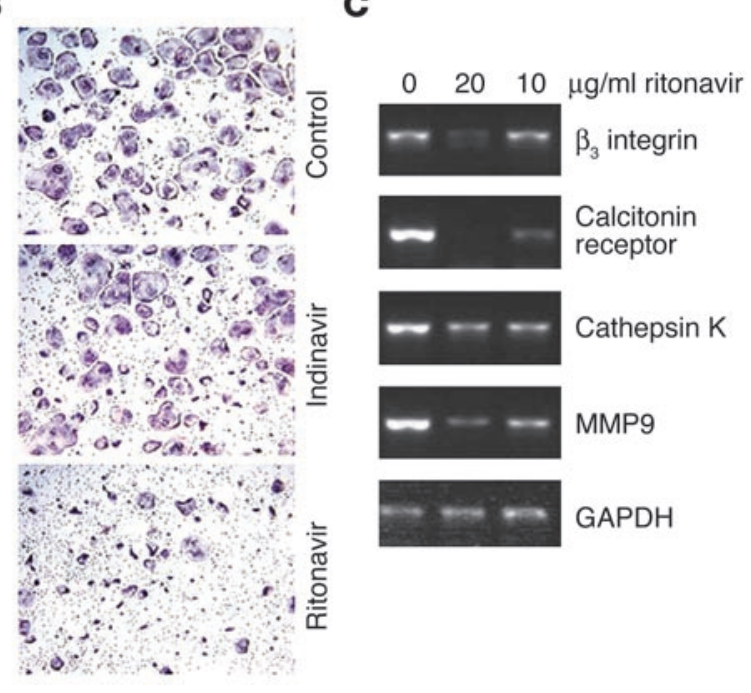

the drug's inhibitory effect on morphological osteoclastogenesis, the PI blunts the expression of a range of osteoclast-defining genes in a parallel fashion (Figure 1C), indicating that the drug acts at an early stage of osteoclast differentiation.

Ritonavir inhibition of osteoclast formation is reversible. To exclude the possibility that ritonavir exerts a toxic effect on osteoclast precursors we asked if its effect on osteoclastogenesis is reversible. Thus, the drug was added at the beginning of osteoclastgenerating cultures at a dose that completely suppresses osteoclast formation $(20 \mu \mathrm{g} / \mathrm{ml})$ and was withdrawn on day 3 , 4 , or 5 . Removal of the drug, up to 5 days after its addition, normalizes osteoclast differentiation by day 7 (Figure 2, A and B). Apoptosis assay of differentiating cells exposed to ritonavir (up to $10 \mu \mathrm{g} / \mathrm{ml}$ ) shows no alterations (data not shown). Cessation of osteoclastogenesis by ritonavir therefore reflects arrested differentiation, rather than toxicity.

\section{Figure 2}

Osteoclastogenic arrest by ritonavir is reversible. (A) Osteoclasts were generated from bone marrow macrophages as in Figure 1 and quantitated by TRAP solution assay. The presence of ritonavir $(20 \mu \mathrm{g} / \mathrm{ml})$ completely suppresses osteoclast formation, while normalization of osteoclast number follows withdrawal of the PI on day 3,4 , and 5. + Ritonavir, continuous ritonavir exposure; - Ritonavir, ritonavir withdrawal. (B) TRAP-stained osteoclasts on day 7 of culture following persistent exposure to ritonavir (left panel) and withdrawal of ritonavir on day 5 (right panel). Magnification, $\times 200$.

\section{Figure 1}

Osteoclastogenesis is impaired by ritonavir but not indinavir. (A) Osteoclasts were generated from bone marrow macrophages stimulated with RANKL and M-CSF for 4 days in the presence of the indicated doses of ritonavir or indinavir. TRAP solution assay quantitation of osteoclast formation shows that the $\mathrm{IC}_{50}$ for ritonavir is near 10 $\mu \mathrm{g} / \mathrm{ml}$. In contrast, cultures exposed to indinavir show no inhibition or enhancement of osteoclast formation. (B) Representative fields of TRAP-stained osteoclasts in the presence of control medium, indinavir $(10 \mu \mathrm{g} / \mathrm{ml})$, and ritonavir $(10 \mu \mathrm{g} / \mathrm{ml})$. Magnification, $\times 100$. (C) Ritonavir dose dependently suppresses osteoclast gene markers determined by RT-PCR analysis of osteoclasts on day 4 culture.

Ritonavir inhibits bone resorption by mature osteoclasts. We next turned to the effect of PIs on the resorptive activity of mature osteoclasts. To this end, ritonavir $(10 \mu \mathrm{g} / \mathrm{ml})$ or indinavir $(10 \mu \mathrm{g} /$ $\mathrm{ml}$ ) was added to cultures after mature osteoclasts had been generated on whale dentine slices in the absence of drugs. Osteoclast number and bone resorption pits were determined 2 days later. As is evident in Figure 3A, ritonavir fails to decrease the number of mature osteoclasts as determined by tartrate-resistant acid phosphatase (TRAP) cytochemistry of whale dentine slices (mean osteoclast number per randomly selected field is $54 \pm 7$ and $62 \pm 12$ in vehicle and ritonavir-treated cultures, respectively), reaffirming the nontoxic effect of the drug on osteoclasts and their precursors. In contrast, pit number, area, and depth are dramatically decreased with ritonavir treatment (Figure 3, A and B). Unlike ritonavir, indinavir fails to alter either the number or functional capacity of mature osteoclasts.
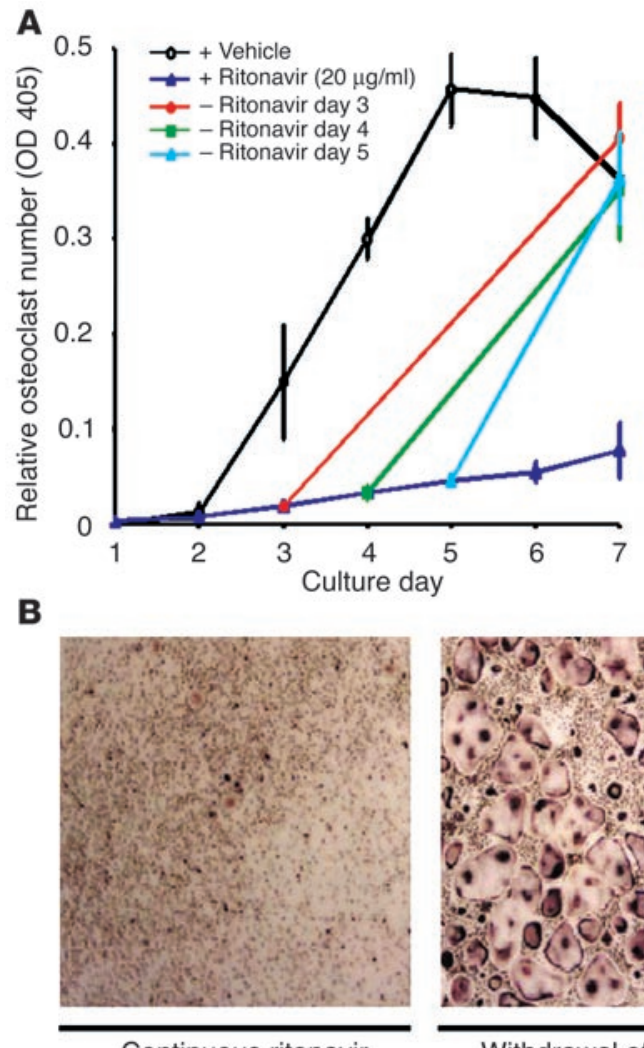

Continuous ritonavir

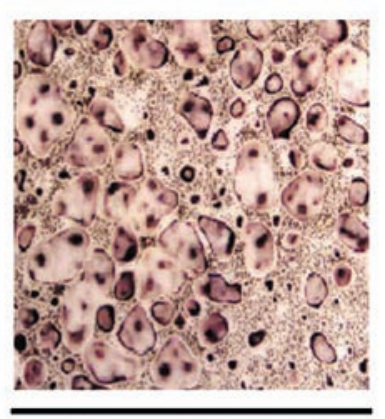

Withdrawal of ritonavir day 5 
A
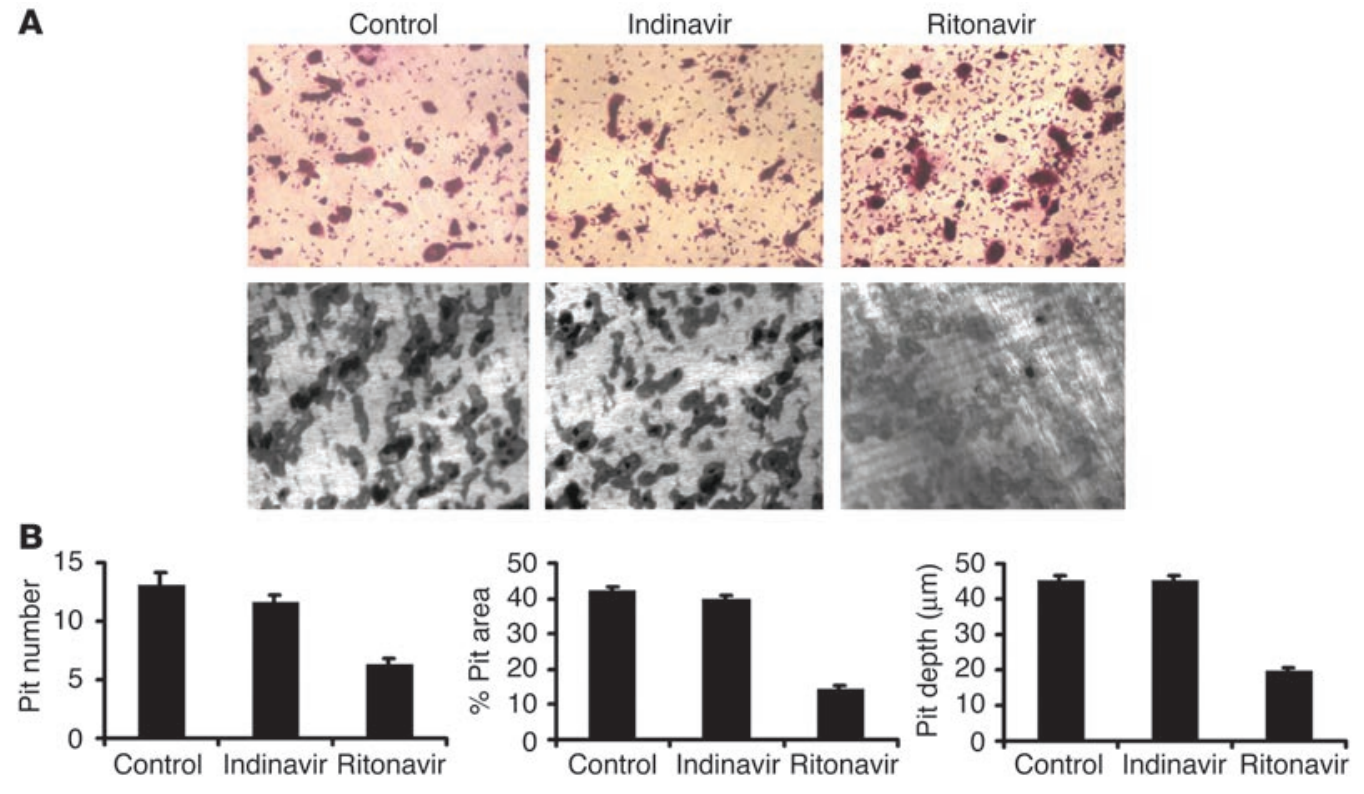

Figure 3

Osteoclast function is impaired by ritonavir. (A) Osteoclasts, generated on whale dentine slices for 3 days by treatment with RANKL and M-CSF, were exposed to control medium, ritonavir $(10 \mu \mathrm{g} / \mathrm{ml})$, or indinavir $(10 \mu \mathrm{g} / \mathrm{ml})$ for an additional 2 days. TRAP-stained dentine slices show no change in osteoclast number with exposure to PIs (top panels). Following cell removal, Coomassie blue staining of dentine slices show decreased bone pits with ritonavir treatment (bottom panels). Magnification, $\times 100$. (B) Ritonavir, but not indinavir, decreases pit number (per $0.36 \mathrm{~mm}^{2}$ ), percentage of pit area, and pit depth (in micrometers) by half (each $P<0.01$ ).

Ritonavir inhibits parathyroid hormone-induced osteoclastogenesis in vivo. Having established that ritonavir arrests osteoclastogenesis in vitro, we asked if the same is obtained in vivo. For these experiments we turned to a murine model of acute osteoclast formation induced by supracalvarial injection of the osteoclastogenic agent, parathyroid hormone $(\mathrm{PTH})(13,14)$, with concomitant intraperitoneal administration of ritonavir. PTH stimulates osteoclastogenesis by targeting directly bone marrow stromal cells to increase the expression of RANKL, thus providing an optimal cellular milieu for osteoclast formation $(3,15)$. Similar to its in vitro effects, ritonavir totally abrogates PTH-induced osteoclast formation when administered systemically (Figure 4, A and B). PTH is also a known inducer of stromal cell proliferation. The increase in stromal cells surrounding the bone matrix is not blocked by ritonavir, as seen in Figure $4 \mathrm{~B}$ and by quantitation (percentage of stromal cell area per total bone marrow in drug-exposed and untreated animals is $75 \pm 12$ and $78 \pm 15$, respectively), arguing that ritonavir inhibits osteoclast formation by targeting the osteoclast precursors rather than impairing PTH action on bone marrow stromal cells.

Ritonavir inhibits RANKL-induced signal transduction. RANKL is essential for both osteoclast differentiation and resorptive function $(2,3)$. Thus, one potential mechanism by which ritonavir impairs osteoclast
A

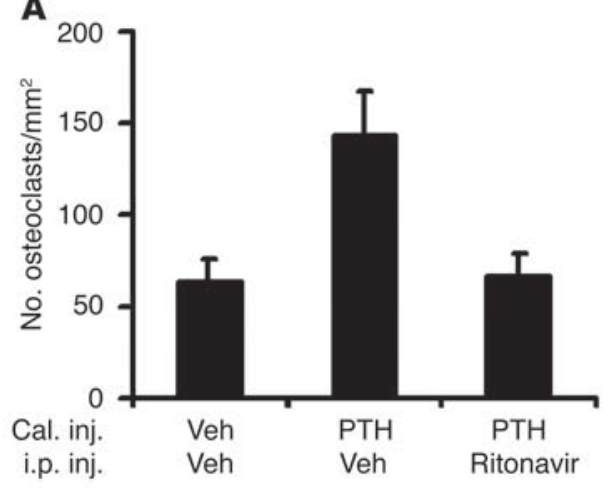

B

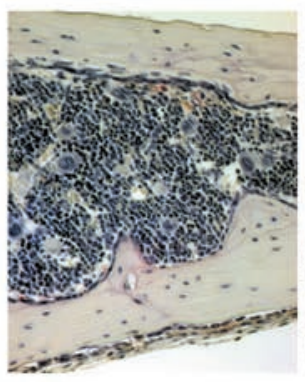

Veh/veh

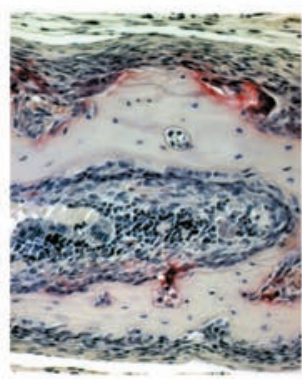

$\mathrm{PTH} /$ veh

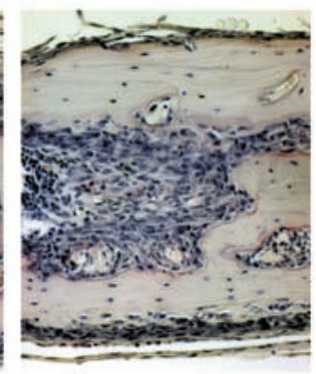

$\mathrm{PTH} /$ ritonavir

Figure 4

Ritonavir blocks PTH-induced osteoclast formation in vivo. (A) Osteoclast number was determined from TRAP-stained histologic sections of calvariae from mice stimulated with PTH or vehicle and intraperitoneally injected with ritonavir or vehicle. Ritonavir abrogates the osteoclast increase stimulated by PTH ( $n=3$ mice per group; $P<0.05)$. Cal. inj., calvarial injection; i.p. inj., intraperitoneal injection; Veh, vehicle. (B) Representative fields of TRAP-stained sections of calvariae show PTH injection fails to induce osteoclast formation in ritonavir-treated mice despite a robust $\mathrm{PTH}$-dependent stromal cell response. Magnification, $\times 250$. 


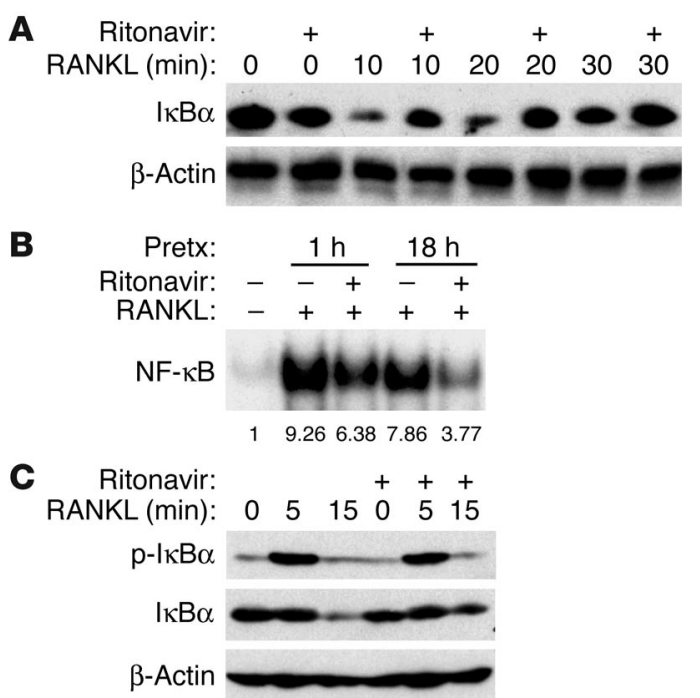

\section{Figure 5}

Ritonavir inhibits RANKL-induced NF- $\mathrm{KB}$ activation. (A) Bone marrow macrophages pretreated with ritonavir $(20 \mu \mathrm{g} / \mathrm{ml})$ or vehicle for 1 hour were stimulated with RANKL for the indicated time points, and protein lysates were prepared for $I \kappa B \alpha$ immunoblot. Ritonavir pretreatment impairs RANKL-induced degradation of $I \kappa B \alpha$. (B) Bone marrow macrophages pretreated with ritonavir $(20 \mu \mathrm{g} / \mathrm{ml})$ for the indicated time were stimulated with RANKL for 15 minutes and evaluated for NF- $\mathrm{KB}$ activation. Ritonavir inhibits NF- $\mathrm{kB}$ activation as assessed by EMSA after 1 hour and after 18 hours of pretreatment (Pretx). Numbers below lanes indicate relative band intensity. (C) Cell lysates prepared as in $\mathbf{A}$ and immunoblotted for phospho-I $\mathrm{KB} \alpha(\mathrm{p}-\mathrm{I} \mathrm{KB} \alpha)$ and $I \kappa \mathrm{B} \alpha$ reveal similar levels of phospho-I $\kappa \mathrm{B} \alpha$ intensity, irrespective of ritonavir pretreatment, despite the failure of ritonavir pretreatment to decrease total $I \kappa B \alpha$ levels. $\beta$-Actin immunoblots confirm similar amounts of cell extracts were analyzed.

formation and function involves the blockade of RANKL-induced signal transduction. To test this possibility, we examined the effect of ritonavir on the known major signaling pathways activated by RANKL ligation of its receptor RANK. Specifically, we analyzed the NF- $\kappa$ B pathway, the Akt axis, and all three MAPK members, namely extracellular signal-related kinases (ERKs; p42/p44), stress activated protein kinase (SAPK/JNK), and p38. Since complete suppression of osteoclast formation is achieved with $20 \mu \mathrm{g} / \mathrm{ml}$ of ritonavir, this concentration was used for all subsequent cell-signaling studies.

$\mathrm{NF}-\kappa \mathrm{B}$ transcription factors are known to play a pivotal role in osteoclast formation as manifested by p50/p52 NF- $\mathrm{\kappa B}$ double-deficient mice, which exhibit osteopetrosis due to a lack of osteoclasts $(16,17)$. Examination of ritonavir's impact on

\section{Figure 6}

Ritonavir inhibits RANKL-induced Akt activation. (A) RAW 264.7 cells pretreated with ritonavir $(2 \mu \mathrm{g} / \mathrm{ml})$ for 1 hour and stimulated with RANKL (+RANKL) for the indicated time points. Phospho-Akt (p-Akt), total Akt (Akt), and phospho-FKHR ( $p$-FKHR) immunoblots were performed on cell extracts. Immunoblots reveal impaired Akt and FKHR phosphorylation with ritonavir pretreatment. (B) Osteoclasts were stimulated with either RANKL or M-CSF for the indicated time points, and cell extracts were immunoblotted for Akt activation. Ritonavir inhibits only RANKL-induced Akt activation but not that stimulated by M-CSF. Total Akt immunoblots confirm that similar amounts of cell extracts were analyzed. $+\mathrm{V}$, vehicle pretreatment; $+\mathrm{R}$, ritonavir pretreatment.
RANKL-induced NF- $\kappa B$ activation reveals impaired degrada-

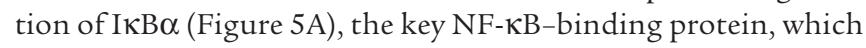
retains the transcription factor in the cytosol. In consequence, the amount of NF- $\mathrm{KB}$ that translocates to the nucleus is also decreased, as determined by electrophoretic mobility shift assay (EMSA; Figure 5B). Of considerable interest, phosphorylation of $\mathrm{I} \kappa \mathrm{B} \alpha$, a prerequisite for its subsequent degradation, is unimpaired by ritonavir (Figure 5C), indicating that ritonavir does not abolish proximal signaling events leading to $I \kappa B \alpha$ phosphorylation but suppresses $I \kappa B \alpha$ degradation.

RANKL activates the Akt signal cascade through a signaling complex containing c-Src and TNF receptor-associated factor 6 (TRAF6), which eventually results in the phosphorylation and activation of Akt (18). To examine RANKL-induced Akt activation in the absence of M-CSF, which is itself a strong Akt activator, we used RAW 264.7 cells, an M-CSF-independent monocytic cell line that can readily differentiate into osteoclasts with RANKL alone. As determined by Western blot analysis, ritonavir impairs RANKL-induced phosphorylation of Akt (Figure 6A). Confirming the functional consequences of this finding, phosphorylation of the forkhead transcription factor (FKHR), a downstream phosphorylation substrate of Akt (19), is also suppressed by the PI (Figure 6A). The impairment of ritonavir-associated Akt activity was similarly observed with primary bone marrow macrophages stimulated with RANKL (data not shown). Given that M-CSF, a cytokine essential for osteoclast survival, is also a potent activator of Akt, we asked whether the ability of ritonavir to block Akt activation is a cytokine-specific event. Thus, we examined Akt signaling in primary macrophage-derived osteoclasts that had been starved of cytokines and serum and then stimulated with RANKL or M-CSF separately. While ritonavir blunts RANKL-induced Akt activation, it does not affect that stimulated by M-CSF (Figure 6B), a result consistent with the in vitro observation that cell survival is unaffected by ritonavir.

Finally, we find that ritonavir has no effect on the three major MAPK signal cascades, ERKs, SAPK/JNK, and p38, activated by RANKL in bone marrow macrophages, osteoclasts, and RAW cells (data not shown). Thus, ritonavir selectively arrests RANKL-induced activation of NF- $\mathrm{KB}$ and Akt, while sparing the MAPK pathways.

Constitutively active $P I 3 K$ rescues ritonavir-induced inbibition of Akt signaling and osteoclast function. To elucidate the molecular basis of Akt pathway impairment by ritonavir, a constitutively active

\section{A}

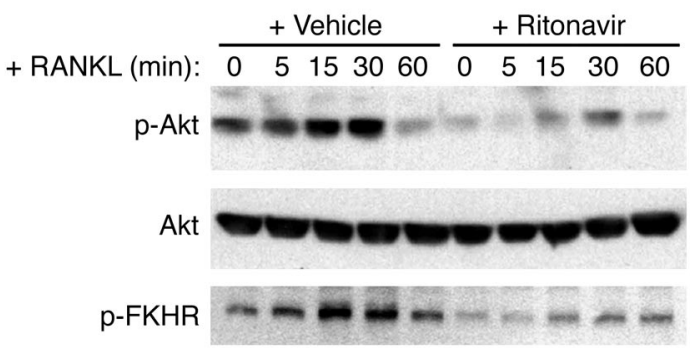

B

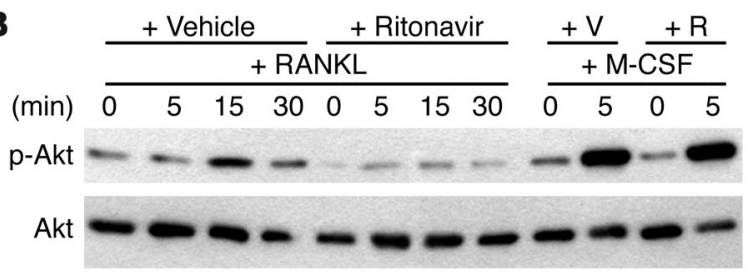




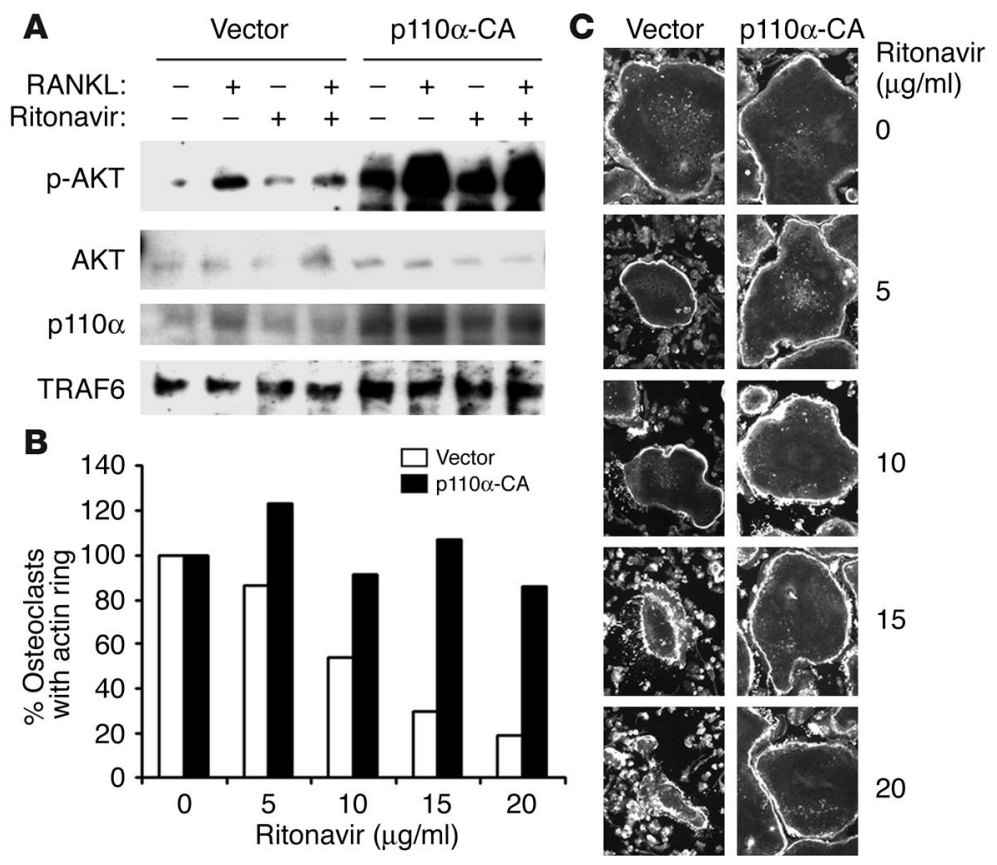

\section{Figure 7}

Introduction of PI3K-CA restores RANKL-induced phosphorylation of Akt and osteoclast actin ring formation in the presence of ritonavir. (A) Retroviral transduction of either vector or PI3K-CA ( $110-\mathrm{CA}$ ) into bone marrow macrophages was followed by 3 days of culture in selection media, M-CSF, and RANKL. After starvation (3 hours) and pretreatment (1 hour) with either vehicle or ritonavir, cells were stimulated with RANKL for 15 minutes. Immunoblots reveal restoration of RANKL-induced Akt phosphorylation when PI3K-CA is introduced. As expected, total PI3K is enhanced as a result of transduction ( $110 \alpha$ blot). TRAF6 Western blots act as a loading control. (B) Percentage of osteoclasts with intact actin rings after ritonavir exposure is quantitated. (C) Osteoclasts, retrovirally transduced with either vector or PI3K-CA, were generated on glass coverslips. After 4 days, cells were exposed to various doses of ritonavir for 2 hours, then processed for immunofluorescence microscopy for $\beta$-actin. Dose-dependent disruption of the characteristic actin ring of the osteoclast cytoskeleton is observed in vector but not PI3K-CA-transduced cells.
(CA) PI3K (PI3K-CA) containing retrovirus was constructed. The activating mutation is generated by the addition of the avian src myristoylation sequence at the $\mathrm{N}$ terminus of the $\mathrm{p} 110 \alpha \mathrm{com}$ ponent of the PI3K. Akt signaling by RANKL in preosteoclasts containing PI3K-CA, but not vector control, are rescued from inhibition by ritonavir (Figure 7A).

Osteoclast actin ring formation and bone resorption is known to be inhibited by PI3K inhibitors such as Wortmannin. Given that ritonavir inhibits osteoclast function (Figure 3, A and B), we determine if the molecular rescue of RANKL-induced Akt signal through the introduction of PI3K-CA can be demonstrated functionally. To this end, mature osteoclasts, transduced with vector or PI3K-CA retroviruses, were exposed to ritonavir and examined by immunofluorescence microscopy for $\beta$-actin after 2 hours of exposure. The reorganization and assembly of actin ring reflects cell polarization and is essential for the resorptive process. In vector control, ritonavir dose dependently disrupts the characteristic actin ring at the osteoclast periphery (Figure $7, \mathrm{~B}$ and $\mathrm{C}$ ). At the highest dose, complete actin ring dissolution is evident. In contrast, actin rings of PI3K-CA-transduced osteoclasts are intact even at the highest dose. These results indicate that ritonavir-induced inhibition of osteoclast function is attributable to the disruption of the PI3K-signaling axis.

Ritonavir inhibits TRAF6 and c-Src recruitment to lipid rafts following RANKL stimulation. The activation of Akt pathway by RANKL has been shown to require the recruitment of TRAF6Src-PI3K complex to the membrane surface by ligand-activated oligomerization of RANK. The ability of PI3K to rescue Akt signaling indicates that ritonavir inhibition of this signaling axis is at or upstream of PI3K. To examine the membrane recruitment of TRAF 6 and c-Src, starved preosteoclasts pretreated with ritonavir were exposed to RANKL for 5 minutes, followed by the isolation of the lipid raft membrane fraction. The recruitment of c-Src and TRAF 6 by RANKL to the lipid raft was inhibited by ritonavir treatment (Figure 8). Interestingly, TRAF2, another
TRAF protein known to be associated with RANK, continues to be recruited to lipid rafts following RANKL simulation, indicating ritonavir specificity in TRAF6 inhibition.

\section{Discussion}

Reports that HIV PI usage is associated with bone demineralization (6-9) prompted us to investigate the direct effect of this class of drugs on the two main cellular determinants of bone mass, osteoblasts and osteoclasts. We noted that HIV PIs have differential effects on osteoblast and osteoclast maturation and function, despite targeting the same catalytic site of the retroviral protease. We reported previously that indinavir, but not ritonavir, is unique in inhibiting osteoblast maturation (10). In contrast, this study demonstrates that ritonavir selectively inhibits osteoclast maturation and function.

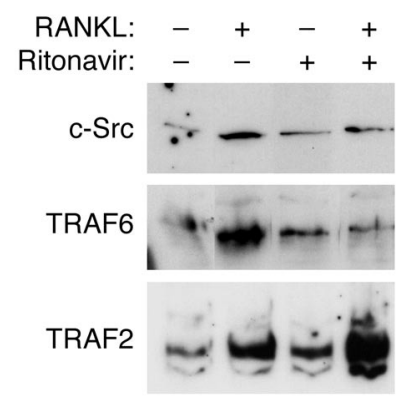

Figure 8

RANKL-induced recruitment of c-Src and TRAF6 to lipid raft component is inhibited by ritonavir treatment. Preosteoclasts generated after 3 days of culture with RANKL and M-CSF were starved for 3 hours, followed by pretreatment with either vehicle or ritonavir (1 hour). Cells were then stimulated with RANKL for 5 minutes, followed by lipid raft isolation. Note the continued recruitment of TRAF2 to lipid rafts with ritonavir treatment, indicating that the inhibition is specific to TRAF6 and c-Src. 
Our in vitro findings reveal that ritonavir directly targets osteoclast precursors, arresting their differentiation in a reversible manner. The PI also directly impacts mature osteoclasts, attenuating their resorptive capacity. This arrest of bone degradation does not reflect cytotoxicity, but rather disruption of the osteoclast cytoskeleton, a complex required for proper polarization and subsequent efficient removal of bone. The in vivo consequences of the antiosteoclastic properties of ritonavir are manifest by the ability of the drug to block PTH-induced osteoclastogenesis.

RANKL is a member of the TNF superfamily with the unique capacity to directly induce osteoclast precursors to differentiate (2). Reflecting its profound impact on osteoclast biology, recent years have witnessed characterization of the major signaling pathways emanating from its receptor, RANK $(12,18,20$, $21)$. We find that ritonavir-mediated inhibition of osteoclastogenesis induced by RANKL reflects blocking of specific components of these intracellular signals. Interestingly, ritonavir inhibits RANKL-induced NF- $\kappa B$ activation, but does so without altering I $\mathrm{K} \mathrm{B} \alpha$ phosphorylation, a process that usually leads to degradation of this NF- $\kappa \mathrm{B}$-binding protein. This failure of $\mathrm{NF}-\kappa \mathrm{B}$ activation in the face of phosphorylated $\mathrm{I} \kappa \mathrm{B} \alpha$ indicates that the PI impacts NF- $\kappa \mathrm{B}$ by preventing the degradation of its major cytosolic-binding protein. In keeping with this conclusion, ritonavir is an established proteasome inhibitor $(22,23)$.

We also find that ritonavir impairs RANKL-induced but not $\mathrm{M}-\mathrm{CSF}-\mathrm{induced}$ Akt activation. Given that RANKL modulates bone resorption, whereas M-CSF affects survival of mature osteoclasts $(2,3)$, this differential signal inhibition is consistent with our in vitro observation of impaired bone resorption without cell death. Furthermore, in mature osteoclasts the ERK signal is responsible for osteoclast survival, while stimulation of the NF- $\kappa B$ pathway activates bone resorption (24). The observation that ritonavir inhibits NF- $\kappa B$ but not the MAPK pathways is in line with the capacity of ritonavir to impair bone resorption in vitro without altering osteoclast number.

We further delineated the specific pathway within the Akt-signaling axis altered by ritonavir, namely the failure of TRAF6 and c-Src to translocate to lipid rafts following RANKL stimulation. The observation is consistent with the ability of a constitutively active form of PI3K, a known downstream signal activated by the TRAF6/c-Src complex, to rescue both Akt signaling and osteoclast cytoskeletal disruption caused by ritonavir. The exact mechanism by which ritonavir inhibits the membrane recruitment of TRAF 6 and c-Src remains to be determined, however.

Reports associating PIs with decreased bone mineral density have assumed all members of this class of drugs are implicated. The fact that ritonavir, but not indinavir, is antiosteoclastic indicates such is not the case. In this regard, others have demonstrated unique properties of these drugs in vitro $(22,25,26)$. Also, clinical studies examining therapy-induced adverse effects have noted a stronger association of hypertriglyceridemia with ritonavir compared with other PIs $(27,28)$.

Recently, Liang et al. proposed that PI-induced hyperlipidemia may be caused by an impaired apoB degradation due to proteasomal inhibition by ritonavir (29). Concerns have been raised regarding the pharmacological relevance of the concentrations used in these experiments $(2 \mu \mathrm{g} / \mathrm{ml}$ as the minimal concentration of ritonavir to exert an effect) $(30,31)$. Our study showed a $50 \%$ inhibition of osteoclast formation at a substantially lower ritonavir concentration $(10 \mu \mathrm{g} / \mathrm{ml})$. Moreover, ritonavir detect- ably blunts osteoclastogenesis at levels as low as $2.5 \mu \mathrm{g} / \mathrm{ml}$ (Figure 1A). Therefore, the plasma concentrations used in this study are within the clinical maximum and minimum values for ritonavir $(11.2 \pm 3.6$ and $3.7 \pm 2.6 \mu \mathrm{g} / \mathrm{ml}$, respectively) and are likely to be pharmacologically relevant.

It is likely that the problem of bone demineralization in HIV-infected patients is complex and multifactorial in origin. Features of HIV itself, especially the increased catabolic rate and associated weight loss, as well as the wasting syndrome seen in advanced AIDS, may contribute to an increased risk of osteopenia in patients. The effect of antiretroviral treatment may also have multiple components, including a direct effect of decreased HIV activity in bone, indirectly because of changes in the immune status or cytokine milieu, or as a direct toxic effect of the drugs used to treat HIV infection. Although we have demonstrated an effect of PIs on bone metabolism, patients receive combination therapies, and the other antiretroviral agents may also have effects that have yet to be elucidated.

Thus, despite the fact that ritonavir and indinavir are designed to arrest the same antiretroviral target, ritonavir is unique in dampening osteoclast function and may be a promising agent in preventing bone loss in HIV-infected individuals. Furthermore, our molecular dissection of signaling pathways specifically impacted by ritonavir may aid in characterizing the mechanisms by which individual PIs prompt idiosyncratic side effects.

\section{Methods}

Reagents. Ritonavir and indinavir were from Abbott Laboratories (Abbott Park, Illinois, USA) and Merck \& Co. Inc. (West Point, Pennsylvania, USA), respectively. All immunoblotting Ab's were from Cell Signaling Technology (Beverly, Massachusetts, USA). Protease inhibitor mixtures used for cell lysis were from Calbiochem-Novabiochem (San Diego, California, USA). The bicinchoninic acid kit for protein determination and ECL kits were obtained from Pierce Biotechnology Inc. (Rockford, Illinois, USA). Recombinant murine M-CSF was from R\&D Systems Inc. (Minneapolis, Minnesota, USA). Murine RANKL was expressed in our laboratory as described previously (32). All other chemicals were obtained from Sigma-Aldrich (St. Louis, Missouri, USA).

Primary cells and cell line. Macrophages/osteoclast precursors and osteoclasts were generated from bone marrow precursors as described (12). RAW 264.7 cells, obtained from American Type Culture Collection (Manassas, Virginia, USA), were maintained as described (33).

Characterization of osteoclasts. Bone marrow macrophages were cultured in 48- or 96-well cell culture dishes in the presence of M-CSF $(10 \mathrm{ng} / \mathrm{ml})$, RANKL (100 ng/ml), and HIV PIs as appropriate, and medium was changed on day 3 . Osteoclast-like cells were characterized by staining for TRAP activity. The number of osteoclasts was assessed by TRAP solution assay as described previously (34).

$R N A$ extraction and amplification by RT-PCR. RNA purification and RT-PCR conditions were as described previously (12). The oligonucleotide primers used are as follows: $\beta_{3}$ integrin, $5^{\prime}$ TTACCCCGTGGACATCTACTA- $3^{\prime}$ and $3^{\prime}$-AGTCTTCCATCCAGGGCAATA- $5^{\prime}$; calcitonin receptor, $5^{\prime}$-CATTCCTGTACTTGGTTGGC-3' and $3^{\prime}$-AGCAATCGACAAGGAGTGAC-5'; cathepsin $\mathrm{K}, 5^{\prime}$-GGAAGAAGACTCACCAGAAGC-3' and $3^{\prime}$ GTCATATAGCCGCCTCCACAG-5'; matrix metalloproteinase 9 (MMP9), 5'-CCTGTGTGTTCCCGTTCATCT-3' and 3'-CGCTGGAATGATCTAAGCCCA-5' ${ }^{\prime}$; GAPDH, $5^{\prime}$-ACTTTGTCAAGCTCATTTCC-3' and $3^{\prime}$-TGCAGCGAACTTTATTGATG-5'. 
Bone resorption. Osteoclasts were generated on whale dentin slices from bone marrow macrophages using conditions described above. After 3 days of culture to generate osteoclasts, PIs were added to the culture for 2 days. At the end of the experiment, cells were TRAP stained and photographed to document cell number. Cells were then removed from the dentin slices with $0.5 \mathrm{M}$ ammonium hydroxide and mechanical agitation. Maximum resorption lacunae depth was measured using a confocal microscope (Microradiance; Bio-Rad Laboratories Inc., Hercules, California, USA) as described (35). For evaluation of pit number and resorbed area, dentin slices were stained with Coomassie brilliant blue and analyzed with light microscopy using Osteomeasure software (Osteometrics Inc., Decatur, Georgia, USA) for quantitation.

Apoptosis assay. Apoptotic cells were assessed using the cell death detection ELISA ${ }^{\text {PLUS }}$ kit (Roche Molecular Biochemicals, Mannheim, Germany). In brief, equal amounts of protein from osteoclastogenic cultures treated with vehicle or ritonavir were incubated with a mixture of Ab's to histones and DNA conjugated, respectively, with biotin and peroxidase. The complex was captured on streptavidin-coated wells, and DNA content was quantitated colorimetrically using a peroxidase substrate.

Immunostaining. Osteoclasts transduced with vector or constitutively active PI3K were generated on glass coverslips using methods described previously (35). After exposing cells to the indicated doses of ritonavir or vehicle control for 2 hours, cells were fixed in $4 \%$ paraformaldehyde, permeabilized in $0.1 \%$ Triton X-100, rinsed in PBS, and immunostained with Alexa 488-phalloidin (Molecular Probes Inc., Eugene, Oregon, USA).

PTH-induced osteoclastogenesis in vivo. To measure responses of murine calvarial bone to PTH, we followed the procedures developed by Boyce and coworkers $(13,14,36)$. Synthetic human $\mathrm{PTH}(1-34)$ was obtained from Bachem California Inc. (Torrance, California, USA) and dissolved in vehicle ( $1 \mathrm{mM} \mathrm{HCl}, 0.1 \% \mathrm{BSA})$. $\mathrm{PTH}$, at $10 \mu \mathrm{g}$ in $25 \mu \mathrm{l}$ or vehicle in the same volume, was injected subcutaneously four times daily for 3 days into the subcutaneous tissue overlying the calvariae, using a Hamilton syringe. During this time, ritonavir $(1 \mathrm{mg})$ or vehicle $(50 \mu \mathrm{l}$ of $25 \%$ ethanol) was injected intraperitoneally twice daily for 3 days. After sacrifice by $\mathrm{CO}_{2}$ narcosis, calvariae were removed intact, soft tissues were gently dissected, and the calvariae were fixed in $10 \%$ phosphate-buffered formalin for 24 hours and further processed as described (14). Samples were analyzed with light microscopy using Osteomeasure software (Osteometrics Inc.). All experiments in vivo were approved by the Washington University Animal Studies Committee.

Cell stimulation and immunoblotting. For NF- $\mathrm{KB}$ and MAPK signaling experiments, in vitro-differentiated bone marrow macrophages were stimulated by adding RANKL in the presence or absence of ritonavir as indicated. For Akt activation, macrophages were cultured in serum and M-CSF-free medium for 24 hours before stimulation. All RANKL-induced signaling in RAW 264.7 cells was performed in nonserum-starved conditions. Ritonavir was added to cultures at $10 \mu \mathrm{g} / \mathrm{ml} 1$ hour prior to stimulation unless otherwise indicated. This dosage level was based on preliminary data, reported here, that the drug does not cause toxicity to cells in the osteoclast lineage. After stimulation, cells were subjected to immunoblotting analysis as described (12).

EMSA. EMSA was performed as described previously (12).

Membrane lipid rafts isolation. Lipid rafts isolation was performed using the detergent-free method of Smart et al. (37).

Statistical analysis. Statistical significance was analyzed using an Excel spreadsheet program (Microsoft Corp., Redmond, Washington, USA) using an unpaired $t$ test.

\section{Acknowledgments}

We thank Deborah Novack for reviewing the manuscript and Paulette Shubert for expert secretarial assistance. This study was supported by the following NIH grants: training grant AR-07033 and F32 fellowship AR-08648 to M.W.-H. Wang; AI-25903 to W.G. Powderly and P. Tebas; AR-32788, AR-46523, and DE-05413 to S.L. Teitelbaum; and AR-46852 and AR-48812 to F.P. Ross.

Received for publication April 25, 2002, and accepted in revised form May 28, 2004.

Address correspondence to: F. Patrick Ross, Department of Pathology and Immunology, Washington University School of Medicine, Campus Box 8118, 660 South Euclid Avenue, St. Louis, Missouri 63110, USA. Phone: (314) 454-8079; Fax: (314) 454-5505; E-mail: rossf@medicine.wustl.edu.
1. Manolagas, S.C. 2000. Birth and death of bone cells: basic regulatory mechanisms and implications for the pathogenesis and treatment of osteoporosis. Endocr. Rev. 21:115-137.

2. Teitelbaum, S.L. 2000. Bone resorption by osteoclasts. Science. 289:1504-1508.

3. Suda, T., et al. 1999. Modulation of osteoclast differentiation and function by the new members of the tumor necrosis factor receptor and ligand families. Endocr. Rev. 20:345-357.

4. Ross, F.P. 2000. RANKing the importance of measles virus in Paget's disease. J. Clin. Invest. 105:555-558.

5. Paton, N.I.J., Macallan, D.C., Griffin, G.E., and Pazianas, M. 1997. Bone mineral density in patients with human immunodeficiency virus infection. Calcif. Tissue Int. 61:30-32.

6. Tebas, P., et al. 2000. Accelerated bone mineral loss in HIV-infected patients receiving potent antiretroviral therapy. AIDS. 14:F63-F67.

7. Moore, A.L., et al. 2001. Reduced bone mineral density in HIV-positive individuals. AIDS. 15:1731-1733.

8. Brown, T., et al. 2002. Bone loss associated with abnormalities in glucose metabolism in HIV patients on protease inhibitors. In Programs and Abstracts of the Ninth Conference of Retroviruses and Opportunistic Infections. Seattle, Washington, USA. 314.

9. Arnsten, J.H., Freeman, R., Santoro, N., Kunins, H., and Schoenbaum, E.E. 2002. Bone mineral density and protease inhibitor use in older HIVinfected women. In Programs and Abstracts of the Ninth Conference on Retroviruses and Opportunistic Infections. Seattle, Washington, USA. 314.

10. Wang, M.W., Wei, S., Teitelbaum, S.L., and Ross, F.P. 2001. The HIV protease inhibitor indinavir uniquely inhibits bone formation (abstract). J. Bone Miner. Res. 16(Suppl.):S372.

11. Teitelbaum, S.L., and Ross, F.P. 2003. Genetic regulation of osteoclast development and function. Nat. Rev. Genet. 4:638-649.

12. Wei, S., Wang, M.W., Teitelbaum, S.L., and Ross, F.P. 2002. Interleukin-4 reversibly inhibits osteoclastogenesis via inhibition of NF- $\mathrm{KB}$ and MAP kinase signaling. J. Biol. Chem. 21:6622-6630.

13. Yates, A.J.P., et al. 1988. Effects of a synthetic peptide of a parathyroid hormone-related protein on calcium homeostasis, renal tubular calcium reabsorption, and bone metabolism in vivo and in vitro in rodents. J. Clin. Invest. 81:932-938.
14. Zhao, W.G., Byrne, M.H., Boyce, B.F., and Krane, S.M. 1999. Bone resorption induced by parathyroid hormone is strikingly diminished in collagenase-resistant mutant mice. J. Clin. Invest. 103:517-524.

15. Ma, Y.L., et al. 2001. Catabolic effects of continuous human PTH (1-38) in vivo is associated with sustained stimulation of RANKL and inhibition of osteoprotegerin and gene-associated bone formation. Endocrinology. 142:4047-4054.

16. Franzoso, G., et al. 1997. Requirement for NF- $\kappa B$ in osteoclast and B-cell development. Genes Dev. 11:3482-3496.

17. Iotsova, V., et al. 1997. Osteopetrosis in mice lacking NF-kappaB1 and NF-kappaB2. Nat. Med. 3:1285-1289.

18. Wong, B.R., et al. 1999. TRANCE, a TNF family member, activates Akt/PKB through a signaling complex involving TRAF6 and c-Src. Mol. Cell. 4:1041-1049.

19. Brunet, A., et al. 1999. Akt promotes cell survival by phosphorylating and inhibiting a Forkhead transcription factor. Cell. 96:857-868.

20. Matsumoto, M., Sudo, T., Saito, T., Osada, A., and Tsujimoto, M. 2000. Involvement of p38 
mitogen-activated protein kinase signaling pathway in osteoclastogenesis mediated by receptor activator of NF- $\mathrm{KB}$ ligand (RANKL). J. Biol. Chem. 275:31155-31161.

21. Kobayashi, N., et al. 2001. Segregation of TRAF6mediated signaling pathways clarifies its role in osteoclastogenesis. EMBO J. 20:1271-1280.

22. Andre, P., et al. 1998. An inhibitor of HIV-1 protease modulates proteasome activity, antigen presentation, and T cell responses. Proc. Natl. Acad. Sci. U. S. A. 95:13120-13124.

23. Schmidtke, G., et al. 1999. How an inhibitor of the HIV-I protease modulates proteasome activity. J. Biol. Chem. 274:35734-35740.

24. Miyazaki, T., et al. 2000. Reciprocal role of ERK and NF- $\mathrm{KB}$ pathways in survival and activation of osteoclasts. J. Cell Biol. 148:333-342.

25. Lenhard, J.M., Weiel, J.E., Paulik, M.A., and Furfine, E.S. 2000. Stimulation of vitamin A(1) acid signaling by the HIV protease inhibitor indinavir. Biochem. Pharmacol. 59:1063-1068.

26. Phenix, B.N., Lum, J.J., Nie, Z., Sanchez-Dardon,
J., and Badley, A.D. 2001. Antiapoptotic mechanism of HIV protease inhibitors: preventing mitochondrial transmembrane potential loss. Blood. 98:1078-1085.

27. Tsiodras, S., Mantzoros, C., Hammer, S., and Samore, M. 2000. Effects of protease inhibitors on hyperglycemia, hyperlipidemia, and lipodystrophy: a 5-year cohort study. Arch. Intern. Med. 160:2050-2056.

28. Fellay, J., et al. 2001. Prevalence of adverse events associated with potent antiretroviral treatment: Swiss HIV Cohort Study. Lancet. 358:1322-1327.

29. Liang, J.S., et al. 2001. HIV protease inhibitors protect apolipoprotein $\mathrm{B}$ from degradation by the proteasome: a potential mechanism for protease inhibitor-induced hyperlipidemia. Nat. Med. 7:1327-1331.

30. Kelleher, A.D., Sewell, A.K., and Price, D.A. 2002 Dyslipidemia due to retroviral protease inhibitors. Nat. Med. 8:308.

31. Sturley, S.L., et al. 2002. Reply to "Dyslipidemia due to retroviral protease inhibitors". Nat. Med.
8:308-309

32. McHugh, K.P., et al. 2000. Mice lacking $\beta 3$ integrins are osteosclerotic because of dysfunctional osteoclasts. J. Clin. Invest. 105:433-440.

33. Wei, S., Wang, M.W.-H., Teitelbaum, S.L., and Ross, F.P. 2001. RANK ligand activates nuclear factor- $\mathrm{\kappa B}$ in osteoclast precursors. Endocrinology. 142:1290-1295.

34. Simonet, W.S., et al. 1997. Osteoprotegerin: a novel secreted protein involved in the regulation of bone density. Cell. 89:309-319.

35. Feng, X., et al. 2001. A Glanzmann's mutation of the $\beta 3$ integrin gene specifically impairs osteoclast function. J. Clin. Invest. 107:1137-1144.

36. Boyce, B.F., Aufdemorte, T.B., Garrett, I.R., Yates, A.J.P., and Mundy, G.R. 1989. Effects of interleukin-1 on bone turnover in normal mice. Endocrinology. 125:1142-1150.

37. Smart, E., Ying, Y., Mineo, C., and Anderson, R. 1995. A detergent-free method for purifying caveolae membrane from tissue culture cells. Proc. Natl. Acad. Sci. U. S. A. 92:10104-10108. 